\title{
Comparative Safety and Effectiveness of Direct-Acting Oral Anticoagulants Versus Warfarin: a National Cohort Study of Nursing Home Residents
}

\author{
Matthew Alcusky, PharmD, PhD ${ }^{7}$, Jennifer Tjia, MD, MS ${ }^{7}$, David D. McManus, MD, ScM ${ }^{1,2}$, \\ Anne L. Hume, PharmD ${ }^{3}$, Marc Fisher, $M D^{4}$, and Kate L. Lapane, $P h D^{7}$
}

\begin{abstract}
'Department of Population and Quantitative Health Sciences, University of Massachusetts Medical School, Worcester, MA, USA; ${ }^{2}$ Department of Medicine, Division of Cardiovascular Medicine, University of Massachusetts Medical School, Worcester, MA, USA; ${ }^{3}$ Department of Pharmacy Practice, College of Pharmacy, University of Rhode Island, Kingston, RI, USA; ${ }^{\circ}$ Department of Neurology, Beth Israel Deaconess Medical Center, Harvard Medical School, Boston, MA, USA.
\end{abstract}

BACKGROUND: Research comparing direct-acting oral anticoagulants (DOACs) to warfarin has excluded nursing home residents, a vulnerable and high-risk population. OBJECTIVE: To compare the safety and effectiveness of DOACs versus warfarin.

DESIGN: New-user cohort study (2011-2016).

PATIENTS: US nursing home residents aged $\geq 65$ years with non-valvular atrial fibrillation enrolled in fee-forservice Medicare for $\geq 6$ months.

EXPOSURES: Initiators of DOACs (2881 apixaban, 1289 dabigatran, 3735 rivaroxaban) were 1:1 propensity matched to warfarin initiators.

MAIN MEASURES: Outcomes included ischemic stroke or transient ischemic attack (i.e., ischemic cerebrovascular event), bleeding (extracranial or intracranial), other vascular events, death, and a composite of all outcomes. Absolute rate differences (RD) and cause-specific hazard ratios (HR) with 95\% confidence intervals (CI) were estimated. Subgroup analyses were performed by alignment of DOAC dosing with labeling.

KEY RESULTS: Median age (84 years), $\mathrm{CHA}_{2} \mathrm{DS}_{2}$-Vasc (5), and ATRIA risk scores (3) were similar across medications. Clinical outcome rates were similar for dabigatran and rivaroxaban users versus warfarin users. However, ischemic cerebrovascular event rates were higher among dabigatran and rivaroxaban users that received reduced dosages without an indication. Overall, apixaban users had higher ischemic cerebrovascular event rates (HR 1.86; 95\% CI 1.00-3.45) and lower bleeding rates (HR 0.66 ; 95\% CI 0.49-0.88), but outcome rates varied by dosing alignment. Mortality rates (per 100 person-years) were lower for apixaban (RDs - 9.30; 95\% CI - 13.18 to 5.42), dabigatran (RDs $-10.79 ; 95 \% \mathrm{CI}-14.98$ to -6.60 ), and rivaroxaban (RDs $-8.92 ; 95 \% \mathrm{CI}-12.01$ to -5.83 ) versus warfarin; composite outcome findings were similar.

Prior Presentations 35th Annual International Conference on Pharmacoepidemiology and Drug Safety, August 28, 2019

Electronic supplementary material The online version of this article (https://doi.org/10.1007/s11606-020-05777-3) contains supplementary material, which is available to authorized users.

Received September 10, 2019

Accepted March 6, 2020

Published online April 6, 2020
CONCLUSIONS: Among US nursing home residents, the DOACs were each associated with lower mortality versus warfarin. Misaligned DOAC dosing was common in nursing homes and was associated with clinical and mortality outcomes. Overall, DOAC users had lower rates of adverse outcomes including mortality compared with warfarin users.

KEY WORDS: comparative effectiveness; anticoagulants; atrial fibrillation; nursing homes.

J Gen Intern Med 35(8):2329-37

DOI: $10.1007 / \mathrm{s} 11606-020-05777-3$

(C) Society of General Internal Medicine 2020

\section{INTRODUCTION}

The RE-LY, ${ }^{1}$ ROCKET-AF, ${ }^{2}$ and ARISTOTLE ${ }^{3}$ trials demonstrated superiority or non-inferiority in safety and effectiveness for each of the direct-acting oral anticoagulants (DOACs) versus warfarin, but excluded nursing home $(\mathrm{NH})$ residents. Observational evidence comparing the safety and effectiveness of warfarin and the DOACs for older adults has also been limited to community-dwelling patients. ${ }^{4-6}$ Generalizability to NH settings is complicated because residents have greater functional limitations, cognitive impairment, polypharmacy, and comorbidities. ${ }^{7}$

To address uncertainty regarding the relative safety and effectiveness of the DOACs and warfarin in the contemporary NH setting, we separately compared new users of apixaban, rivaroxaban, and dabigatran to new users of warfarin in a national cohort of NH residents with atrial fibrillation. Motivated by earlier reports describing deviation from labeled dosing, ${ }^{7,} 8$ we also examined heterogeneity in estimates across strata defined by alignment (or misalignment) of DOAC dosing with labeling recommendations.

\section{METHODS}

\section{Data Sources}

Data were obtained through a data use agreement with the Centers for Medicare and Medicaid Services. The Master 
Beneficiary Summary File contained vital status and enrollment in Medicare and Medicaid. Medicare Part A provided inpatient and skilled nursing facility (SNF) records. Medication dispensing records and drug characteristics were sourced from the Medicare Part D Event and Characteristics Files. The Minimum Data Set (MDS) 3.0, composed of (previously validated $)^{9}$ national data collected through mandatory assessments, provided information on SNF and long-stay residents in Medicare/Medicaid-certified NHs (96\% of US NHs). The UMassMed IRB approved this study (H00015376).

\section{Study Design}

A retrospective cohort study compared $\mathrm{NH}$ residents initiating apixaban, rivaroxaban, or dabigatran to warfarin initiators during the period July 01, 2011, to December 31, 2016. The index date was the first observed dispensing of an oral anticoagulant. Indexing of new DOAC users began in the month following marketing of apixaban (12/2012) and rivaroxaban (11/2011). Follow-up continued until a study outcome; anticoagulant discontinuation (treatment gap $>14$ days); anticoagulant switch; end of Medicare Parts A, B, and D fee-forservice enrollment; or end of the study period (12/31/2016).

\section{Source Population}

The population included residents of US NHs $\geq 65$ years of age and diagnosed with non-valvular atrial fibrillation who newly initiated a DOAC or warfarin. Included residents had $\geq$ 6 months of pre-index Medicare fee-for-service enrollment and at least one diagnosis for atrial fibrillation, atrial flutter, or dysrhythmia on Part A or MDS 3.0 records during the preindex year (Supplemental Table 1). Because medications in hospitals, SNF, or hospice settings are not included in Part D data, we excluded residents in these settings on the index date. We also excluded residents with another indication for anticoagulant initiation based on an inpatient diagnosis of venous thromboembolism (VTE), valvular disease, or total hip/knee replacement during the baseline 6 months. Residents with cancer or in a coma were also excluded.

\section{Anticoagulant Use}

We operationalized anticoagulant use to evaluate specific anticoagulants (warfarin, apixaban, dabigatran, rivaroxaban). New users were residents initiating one of these medications without prior use of an oral anticoagulant in the preceding 6 months. The date of the first oral anticoagulant dispensing was the index date. Following the index dispensing, an astreated approach was implemented using fill dates and number of days supplied from Part D claims to determine if residents remained exposed to the index medication on each day of follow-up. The end of treatment was assigned once the supply from the most recent medication fill was depleted and a gap in treatment of $>14$ days occurred. Because DOACs are available in two dosages, we categorized residents as initiating standard or reduced doses. Rivaroxaban $10 \mathrm{mg}$ users were considered less than standard dose users not aligned with labeling.

\section{Outcomes}

Primary time-to-event outcomes included ischemic cerebrovascular event (ischemic stroke or transient ischemic attack (TIA)), intracranial or extracranial (all non-cerebral sites) bleeding, and a composite net clinical benefit outcome comprising ischemic stroke, TIA, intracranial bleeding, extracranial bleeding, venous thromboembolism (VTE), acute myocardial infarction (AMI), systemic embolism, and all-cause mortality. Each of the components of the net clinical benefit outcome was evaluated as secondary outcome. All clinical outcomes were operationalized from diagnoses on hospitalization records using validated ICD-9 code-based algorithms (Supplemental Table 1), ${ }^{10-13}$ which were converted to ICD-10 using General Equivalence Mapping. ${ }^{14}$ All outcomes were evaluated for the maximum duration of follow-up.

\section{Covariates}

Covariates included sociodemographics, the $\mathrm{CHA}_{2} \mathrm{DS}_{2}$ VASc $^{15}$ and ATRIA ${ }^{16}$ risk scores, and their components, hospitalizations, medication use, cognitive impairment, and functioning in activities of daily living (ADLs). Baseline hospitalizations for clinical events were recorded (Supplemental Table 1). ${ }^{10,12}$ The number of hospitalizations and unique medications during the pre-index 6 months was summed. Residents were classified as users of medication classes (antiplatelets, non-steroidal anti-inflammatory drugs, statins, angiotensin-converting enzyme inhibitors and angiotensin receptor blockers, selective serotonin reuptake inhibitors) associated with study outcomes ${ }^{17-21}$ if $>1$ Part D claim was present during the pre-index 6 months.

Comorbid clinical conditions were operationalized using information from MDS 3.0 assessments with the exception of renal functioning, which was categorized as no impairment, chronic renal insufficiency without end-stage disease or dialysis, ${ }^{22}$ end-stage renal disease (MDS item I1500) without dialysis, and dialysis (MDS item O0100J2). Cognitive status was categorized using the MDS 3.0 Cognitive Function Scale. ${ }^{23}$ Functional limitations were summarized using the ADL score (range 0-16, higher scores indicate greater limitation). ${ }^{24}$

\section{Statistical Analysis}

We developed three separate cohorts for each DOAC using nearest neighbor propensity score matching (caliper 0.05 ) to assemble comparable groups of warfarin initiators. Propensity score estimation included the above-described covariates (each associated with $\geq 1$ outcome), ${ }^{17-21,} 25-27$ avoiding bias from inclusion of variables only associated with exposure. ${ }^{28-}$ ${ }^{30}$ Matching was performed within index year to identify a 
comparison group that reflected prevailing real-world treatment conditions during a given period. After matching, all characteristics with sufficient prevalence in the cohort $(\geq 5 \%)$ to represent potential confounding threats were well-balanced (standardized difference $<0.10)^{31}$

Incidence rates were calculated for all primary and secondary outcomes. Cox proportional hazards models estimated cause-specific hazard ratios to account for competing risks of other study outcomes. To avoid censoring patients who experienced a clinical event and then died shortly thereafter, residents were not censored for clinical events in all-cause mortality analyses. More than half of NHs contributed a single resident; Cox proportional hazards frailty models which accounted for state-level clustering did not change estimates. Dose was examined in combination with renal function as a source of heterogeneity. Pre-specified analyses were performed within subgroups defined by DOAC dose alignment with labeling (Supplemental Table 2). ${ }^{32-34}$ To evaluate channeling bias in the early DOAC post-approval period, ${ }^{35}$ we conducted stratified analyses by halves of the study period. To examine the effects of the drug interaction between anticoagulants and antiplatelets, stratified analyses were performed. The proportional hazards assumption was evaluated graphically (log-log plots and plots of Schoenfeld residuals) and satisfied for all models.

\section{Sensitivity Analyses}

To examine residual confounding, hospitalizations for pneumonia and chronic obstructive pulmonary disease (COPD)

Table 1 Characteristics of Residents Treated with Apixaban, Dabigatran, Rivaroxaban, and Matched Warfarin Users

\begin{tabular}{|c|c|c|c|c|c|c|}
\hline & \multicolumn{2}{|c|}{ Apixaban cohort } & \multicolumn{2}{|c|}{ Dabigatran cohort } & \multicolumn{2}{|c|}{ Rivaroxaban cohort } \\
\hline & $\begin{array}{l}\text { Apixaban } \\
n=2881\end{array}$ & $\begin{array}{l}\text { Warfarin } \\
n=2881\end{array}$ & $\begin{array}{l}\text { Dabigatran } \\
n=1289\end{array}$ & $\begin{array}{l}\text { Warfarin } \\
n=1289\end{array}$ & $\begin{array}{l}\text { Rivaroxaban } \\
n=3735\end{array}$ & $\begin{array}{l}\text { Warfarin } \\
n=\mathbf{3 7 3 5}\end{array}$ \\
\hline \multicolumn{7}{|l|}{ Demographics } \\
\hline Age in years, median (Q1, Q3) & $84(77,89)$ & $84(76,89)$ & $83(77,89)$ & $83(77,89)$ & $84(77,89)$ & $84(77,89)$ \\
\hline Women $(\%)$ & 68.5 & 67.8 & 67.2 & 69.7 & 69.3 & 68.2 \\
\hline Non-white race/ethnicity $(\%)$ & 15.6 & 15.1 & 13.8 & 13.0 & 15.0 & 15.0 \\
\hline Enrolled in Medicaid (\%) & 68.5 & 70.3 & 74.9 & 72.2 & 72.1 & 71.8 \\
\hline \multicolumn{7}{|l|}{ Hospital admissions in prior year (\%) } \\
\hline 1 & 36.8 & 37.9 & 33.4 & 31.3 & 36.1 & 36.4 \\
\hline $2-3+$ & 33.4 & 32.7 & 30.1 & 31.0 & 30.6 & 28.6 \\
\hline Ischemic stroke & 12.7 & 12.8 & 12.6 & 11.4 & 10.6 & 10.6 \\
\hline Extracranial or intracranial bleed & 1.8 & 1.8 & 1.5 & 1.8 & 1.3 & 1.5 \\
\hline $\begin{array}{l}\text { Time since first observed nursing } \\
\text { home entry, median (Q1, Q3) }\end{array}$ & $\begin{array}{l}588(103 \\
1319)\end{array}$ & $609(114,1287)$ & $325(108,618)$ & $302(100,617)$ & $\begin{array}{l}543(118 \\
1073)\end{array}$ & $\begin{array}{l}585(120 \\
1103)\end{array}$ \\
\hline $\begin{array}{l}\text { Unique medications, median } \\
\text { (Q1, Q3) }\end{array}$ & $21(13,31)$ & $22(13,32)$ & $17(11,25)$ & $16(10,24)$ & $21(13,30)$ & $21(13,31)$ \\
\hline \multicolumn{7}{|l|}{ DOAC dose $(\%)$} \\
\hline Less than standard & 50.3 & NA & 42.1 & NA & 59.4 & NA \\
\hline \multicolumn{7}{|l|}{ Select medications (\%) } \\
\hline NSAID & 35.5 & 36.4 & 28.2 & 25.6 & 35.1 & 36.7 \\
\hline Antiplatelet & 25.0 & 23.7 & 23.0 & 22.7 & 22.7 & 22.2 \\
\hline Statin & 59.5 & 60.5 & 49.4 & 45.5 & 54.9 & 55.5 \\
\hline SSRI & 47.2 & 48.2 & 45.6 & 46.2 & 48.9 & 49.3 \\
\hline ACE inhibitor or ARB & 62.0 & 62.4 & 59.4 & 57.0 & 60.9 & 60.6 \\
\hline \multicolumn{7}{|l|}{ Comorbidities $(\%)$} \\
\hline Diabetes & 39.5 & 37.5 & 37.2 & 35.7 & 35.3 & 35.7 \\
\hline Heart failure & 35.8 & 34.8 & 36.9 & 36.5 & 33.4 & 33.6 \\
\hline Hypertension & 85.9 & 85.7 & 84.2 & 83.4 & 83.9 & 84.0 \\
\hline Coronary artery disease & 29.9 & 27.5 & 29.3 & 28.1 & 26.4 & 25.6 \\
\hline Anemia & 29.5 & 28.8 & 27.4 & 26.4 & 28.8 & 29.4 \\
\hline Fall history & 16.4 & 17.8 & 17.1 & 15.4 & 19.0 & 19.7 \\
\hline Stroke & 22.0 & 21.4 & 24.7 & 23.5 & 22.5 & 21.7 \\
\hline \multicolumn{7}{|l|}{ Renal impairment } \\
\hline Chronic renal insufficiency & 22.1 & 23.4 & 16.8 & 16.1 & 19.4 & 20.4 \\
\hline End-stage renal disease & 13.7 & 13.8 & 11.5 & 12.7 & 11.5 & 12.5 \\
\hline Dialysis & 3.1 & 3.7 & $\begin{array}{l}\text { Suppressed per } \\
\text { DUA }\end{array}$ & $\begin{array}{l}\text { Suppressed per } \\
\text { DUA }\end{array}$ & 0.5 & 0.6 \\
\hline History of pneumonia & 6.0 & 7.2 & 5.5 & 5.8 & 7.0 & 6.0 \\
\hline Chronic lung disease & 26.5 & 27.3 & 26.8 & 29.6 & 27.9 & 28.0 \\
\hline $\begin{array}{l}\mathrm{CHA}_{2} \mathrm{DS}_{2} \text {-Vasc risk score, median } \\
(\mathrm{Q} 1, \mathrm{Q} 3)\end{array}$ & $5(4,6)$ & $5(4,6)$ & $5(4,6)$ & $5(4,6)$ & $5(4,6)$ & $5(4,6)$ \\
\hline $\begin{array}{l}\text { ATRIA bleeding risk score, median } \\
\text { (Q1, Q3) }\end{array}$ & $3(3,6)$ & $3(3,6)$ & $3(3,6)$ & $3(3,6)$ & $3(3,6)$ & $3(3,6)$ \\
\hline \multicolumn{7}{|l|}{ Cognitive skills $(\%)$} \\
\hline Mildly impaired & 26.2 & 25.7 & 27.5 & 26.5 & 26.5 & 25.6 \\
\hline Moderately to severely impaired & 32.8 & 33.0 & 33.1 & 34.7 & 37.0 & 37.0 \\
\hline $\begin{array}{l}\text { ADL score }(0-16), \text { median } \\
(\mathrm{Q} 1, \mathrm{Q} 3)\end{array}$ & $10(7,11)$ & $10(7,11)$ & $9(6,11)$ & $9(7,11)$ & $10(7,12)$ & $10(8,12)$ \\
\hline Life expectancy $>6$ months & 99.5 & 99.7 & $>99.5$ & $>99.5$ & $>99.0$ & $>99.0$ \\
\hline
\end{tabular}

ADLs activities of daily living, NSAID non-steroidal anti-inflammatory drugs, SSRI selective serotonin reuptake inhibitor, ACE angiotensin-converting enzyme, ARB angiotensin receptor blocker, DUA data use agreement, DOAC direct-acting oral anticoagulant 
were evaluated as falsification outcomes not expected to be associated with treatment. $^{36,37}$

\section{RESULTS}

Among 3422 apixaban, 3758 rivaroxaban, and 1289 dabigatran initiators, $84 \%, 99 \%$, and $100 \%$ were matched to warfarin initiators. The matched apixaban, rivaroxaban, and dabigatran cohorts were drawn from 2599, 3065, and 1549 facilities, respectively.

\section{Resident Characteristics}

Resident characteristics were summarized by treatment for the eligible population ( $n=21,346$; Supplemental Table 3) and within matched cohorts (Table 1). Warfarin and DOAC users within each cohort had median $\mathrm{CHA}_{2} \mathrm{DS}_{2}$-Vasc risk scores of 5 (Q1 4, Q3 6) and ATRIA risk scores of 3 (Q1 3, Q3 6).

\section{Follow-up}

The median duration of follow-up in the apixaban cohort was 137 (Q1 45, Q3 326) days for apixaban and 124 (Q1 40, Q3 285) days for warfarin users, during which time 663 events and 767 events occurred over 1792 and 1602 person-years, respectively. Median follow-up was 134 (Q1 44, Q3 162) days for dabigatran and 212 (Q1 57, Q3 580) days for matched warfarin users, during which time 372 and 571 events occurred during 1153 and 1384 person-years, respectively. Median follow-up was 139 (Q1 42, Q3 374) days for rivaroxaban and 147 (Q1 44, Q3 376) days for warfarin users, during which time 1049 and 1223 events occurred over 2710 and 2722 person-years, respectively. The most common reason for censoring was end of the study in the apixaban cohort and medication discontinuation in the dabigatran and rivaroxaban cohorts (Supplemental Table 4).

\section{Apixaban Versus Warfarin}

The incidence of ischemic stroke/TIA was 1.67 events/ 100 person-years among apixaban users and 0.94 events/ 100 person-years among warfarin users (Fig. 1; Table 2). Bleeding (intracranial and extracranial) rates were 4.35 and 6.74 events per 100 person-years among apixaban and warfarin users, respectively.

Hazard ratios comparing apixaban to warfarin were 1.86 (95\% CI 1.00-3.45) for ischemic stroke/TIA and 0.66 (0.490.88 ) for bleeding (Table 3 ). The mortality hazard ratio closely resembled the composite hazard ratio (HR 0.79; 95\% CI 0.710.88). Estimates for individual clinical components were directionally aligned with the composite outcomes, with modest variation in magnitude (Supplemental Table 5).

In analyses exploring heterogeneity by alignment of dosing with labeling recommendations, hazard ratios comparing bleeding rates between apixaban and warfarin consistently favored apixaban except in the subgroup receiving standard dose apixaban in the presence of an indication for dose reduction (Fig. 2; Supplemental Table 6). Although point estimates for ischemic stroke/TIA consistently favored warfarin, the number of events was small in each subgroup. Mortality (and composite) rates were lower among aligned standard dose apixaban users and among low dose apixaban users without an indication for dose reduction.

In analyses by index year (Table 3 ), point estimates favored apixaban for all outcomes among those initiating anticoagulants in 2013-2014. Associations were attenuated, or in the case of ischemic stroke/TIA, reversed among those initiating during 2015-2016. Stratified analyses by antiplatelet use suggested heterogeneity may exist in the treatment-outcome association (Supplemental Table 7). Associations between treatment and the falsification outcomes pneumonia and COPD were not indicative of strong residual cofounding (Supplemental Table 8). Modest heterogeneity by sex and race/ethnicity was observed for stroke/TIA only (Supplemental Table 9).

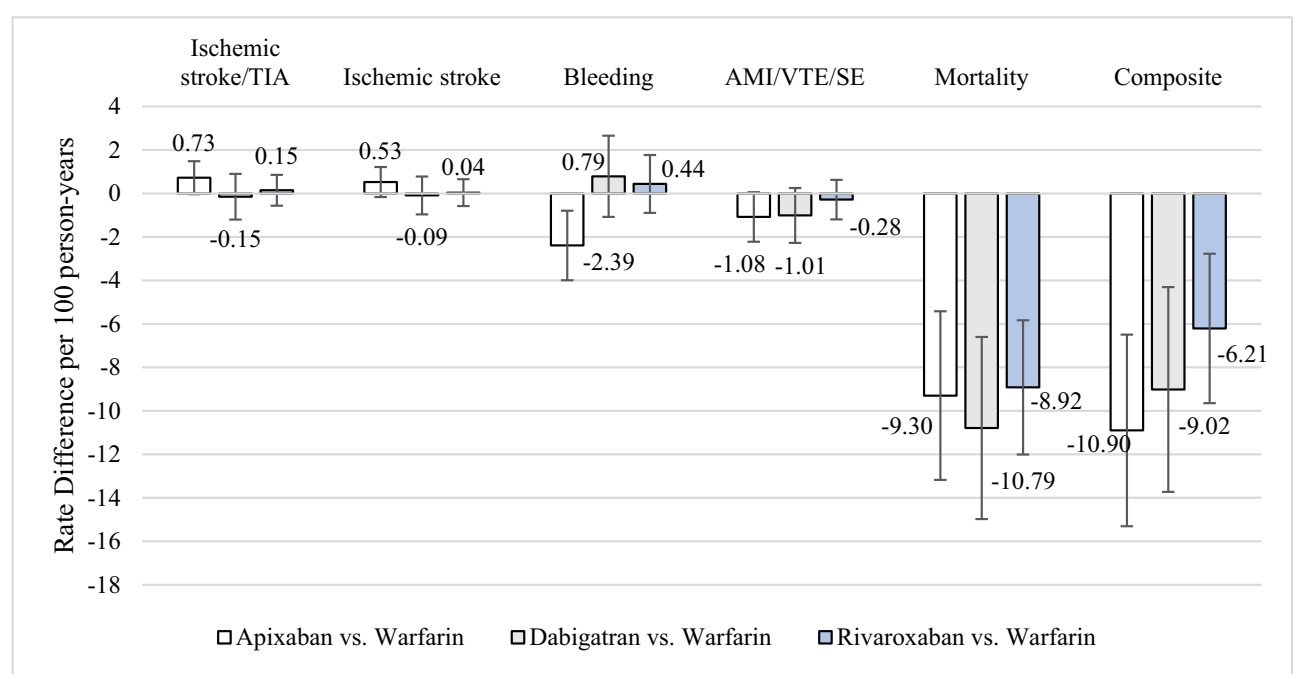

Figure 1 Absolute differences in clinical event, mortality, and composite outcome rates between direct-acting oral anticoagulant and warfarin users. TIA, transient ischemic attack; AMI, acute myocardial infarction; VTE, venous thromboembolism; SE, systemic embolism. 
Table 2 Number of Events and Incidence Rates by Anticoagulant in Matched Cohorts of DOAC and Warfarin Users

\begin{tabular}{|c|c|c|c|c|c|}
\hline & No. of events & Events/100 PYs & No. of events & Events/100 PYs & Rate difference $(95 \% \mathrm{CI})$ \\
\hline & \multicolumn{2}{|c|}{ Apixaban $n=2881$} & \multicolumn{2}{|c|}{ Warfarin $n=2881$} & Apixaban vs. warfarin \\
\hline Ischemic stroke/TIA & 30 & 1.67 & 15 & 0.94 & $0.73(-0.03$ to 1.49$)$ \\
\hline Ischemic stroke & 24 & 1.34 & 13 & 0.81 & $0.53(-0.16$ to 1.22$)$ \\
\hline Bleeding & 78 & 4.35 & 108 & 6.74 & $-2.39(-3.99$ to -0.79$)$ \\
\hline AMI/VTE/SE & 41 & 2.29 & 54 & 3.37 & $-1.08(-2.22$ to 0.06$)$ \\
\hline Mortality $\dagger$ & 554 & 30.7 & 645 & 40.0 & $-9.30(-13.18$ to -5.42$)$ \\
\hline \multirow[t]{2}{*}{ Composite } & 669 & 37.0 & 767 & 47.9 & $-10.90(-15.31$ to -6.49$)$ \\
\hline & Dabi & $n=1289$ & War & $=1289$ & Dabigatran vs. Warfarin \\
\hline Ischemic stroke/TIA & 20 & 1.73 & 26 & 1.88 & $-0.15(-1.20$ to 0.90$)$ \\
\hline Ischemic stroke & 14 & 1.21 & 18 & 1.30 & $-0.09(-0.96$ to 0.78$)$ \\
\hline Bleeding & 70 & 6.07 & 73 & 5.28 & $0.79(-1.08$ to 2.66$)$ \\
\hline AMI/VTE/SE & 25 & 2.17 & 44 & 3.18 & $-1.01(-2.27$ to 0.25$)$ \\
\hline Mortality $\dagger$ & 283 & 24.3 & 496 & 35.09 & $-10.79(-14.98$ to -6.60$)$ \\
\hline \multirow[t]{2}{*}{ Composite } & 372 & 32.25 & 571 & 41.27 & $-9.02(-13.73$ to -4.31$)$ \\
\hline & Rivar & $n=3735$ & War & $=3735$ & Rivaroxaban vs. warfarin \\
\hline Ischemic stroke/TIA & 50 & 1.84 & 46 & 1.69 & $0.15(-0.56$ to 0.86$)$ \\
\hline Ischemic stroke & 37 & 1.36 & 36 & 1.32 & $0.04(-0.58$ to 0.66$)$ \\
\hline Bleeding & 175 & 6.46 & 164 & 6.02 & $0.44(-0.89$ to 1.77$)$ \\
\hline AMI/VTE/SE & 76 & 2.80 & 84 & 3.08 & $-0.28(-1.19$ to 0.63$)$ \\
\hline Mortality $\dagger$ & 824 & 30.15 & 1052 & 38.07 & $-8.92(-12.01$ to -5.83$)$ \\
\hline Composite & 1049 & 38.70 & 1223 & 44.91 & $-6.21(-9.65$ to -2.77$)$ \\
\hline
\end{tabular}

Matched on propensity scores that were estimated using the following variables: age, sex, cognitive status, functioning in activities of daily living, $\mathrm{CHA}_{2} \mathrm{DS} \mathrm{S}_{2}$-Vasc score, ATRIA score, number of recent hospitalizations, recent ischemic stroke hospitalization, recent TIA hospitalization, recent AMI hospitalization, recent systemic embolism hospitalization, recent extracranial bleed hospitalization, recent intracranial hemorrhage hospitalization, renal functioning, recent fall, hip fracture, coronary artery disease, peripheral vascular disease, anemia, diabetes, heart failure, hypertension, stroke, hemiplegia, aphasia, use of ACE inhibitors/ARBs, NSAID use, antiplatelet use, statin use, selective serotonin reuptake inhibitor use, and number of unique medications

$\$ 40$ apixaban (7.2\% of those who died) and 58 (8.9\%) warfarin users experienced a clinical event prior to death; 26 (9.2\%) dabigatran and 68 (13.7\%) warfarin users experienced a clinical event prior to death; 76 (9.2\%) rivaroxaban users and 123 (11.7\%) warfarin users experienced a clinical event prior to death

TIA transient ischemic attack, AMI acute myocardial infarction, VTE venous thromboembolism, SE systemic embolism, DOAC direct-acting oral anticoagulant, ACE angiotensin-converting enzyme, ARBs angiotensin receptor blockers, NSAIDS non-steroidal anti-inflammatory drugs

\section{Dabigatran Versus Warfarin}

The incidence of ischemic stroke/TIA among dabigatran and warfarin users was 1.73 and 1.88 events per 100 person-years, respectively. Bleeding rates per 100 person-years were 6.07 (dabigatran) and 5.28 (warfarin). Hazard ratios did not suggest a meaningful difference in the rate of either outcome. Mortality and composite event rates were lower among dabigatran users.

Although confidence intervals were wide, point estimates for ischemic stroke/TIA favored dabigatran across dosing subgroups with the exception of those receiving less than standard dosing without an indication (Fig. 2, Supplemental Table 6). Bleeding rates were comparable for dabigatran and warfarin users across dosing subgroups with the exception of the group receiving standard doses in the presence of an indication for dose reduction. Mortality and composite rates were lower among dabigatran users in each dosing subgroup except for those receiving standard doses in the presence of an indication for dose reduction.

\section{Rivaroxaban Versus Warfarin}

The incidence of ischemic stroke/TIA was 1.84 events/ 100 person-years among rivaroxaban and 1.69 events/ 100 person-years among warfarin users. Bleeding rates were 6.46 (rivaroxaban) and 6.02 (warfarin) events per 100 personyears. Intracranial hemorrhage, mortality, and composite event rates were lower among rivaroxaban users. As in the apixaban cohort, heterogeneity in the incidence of ischemic and bleeding events was observed between warfarin initiators in the first and second halves of the study period (Table 3 ).

In subgroup analyses by dosing alignment (Fig. 2, Supplemental Table 6), mortality and composite event rates were lower among standard dose rivaroxaban users but not among low dose rivaroxaban users, regardless of indication. Bleeding rates were higher among low dose rivaroxaban users with an indication for dose reduction, while ischemic stroke/TIA rates were higher among low dose rivaroxaban users without an indication.

\section{DISCUSSION}

In this national study of US NH residents, apixaban, rivaroxaban, and dabigatran were associated with lower mortality rates compared with warfarin. Treatment-outcome associations for clinical endpoints varied between DOACs. Heterogeneity was observed across dosing subgroups and over the study period. In aggregate, the results of this first investigation of the comparative effectiveness of the DOACs versus warfarin among $\mathrm{NH}$ residents suggested that DOAC use was associated with lower rates of adverse outcomes including mortality compared with warfarin.

Despite the large burden of vascular risk factors in the $\mathrm{NH}$ population, we observed ischemic stroke rates generally consistent with anticoagulants in BAFTA and the DOAC trials. ${ }^{1-3 \text {, }}$ 
Table 3 Incidence Rates and Results of Cox Proportional Hazards Models Comparing Matched DOAC and Warfarin Groups Overall and by Time Period

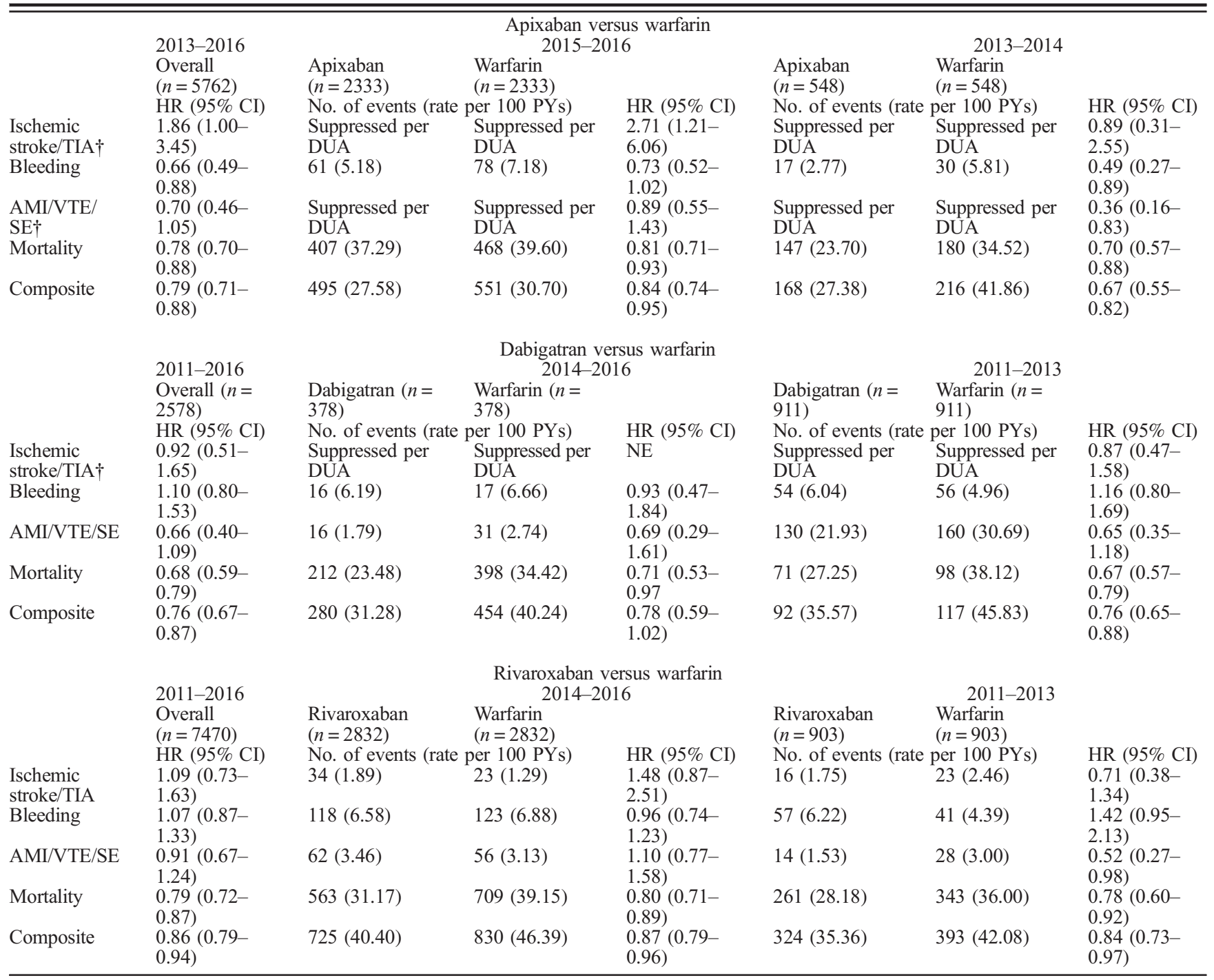

Matched on propensity scores that were estimated using the following variables: age, sex, cognitive status, functioning in activities of daily living, $\mathrm{CHA}_{2} \mathrm{DS} \mathrm{S}_{2}$-Vasc score, ATRIA score, number of recent hospitalizations, recent ischemic stroke hospitalization, recent TIA hospitalization, recent AMI hospitalization, recent systemic embolism hospitalization, recent extracranial bleed hospitalization, recent intracranial hemorrhage hospitalization, renal functioning, recent fall, hip fracture, coronary artery disease, peripheral vascular disease, anemia, diabetes, heart failure, hypertension, stroke, hemiplegia, aphasia, use of ACE inhibitors/ARBs, NSAID use, antiplatelet use, statin use, selective serotonin reuptake inhibitor use, and number of unique medications

†Cell sizes suppressed so that no cell was $<11$ per data use agreement

TIA transient ischemic attack, AMI acute myocardial infarction, VTE venous thromboembolism, SE systemic embolism, NE not estimable, HR hazard ratio, CI confidence interval, DOAC direct-acting oral anticoagulant, DUA data use agreement

${ }^{38}$ Rates of major bleeding, a more stringent definition than our hospitalization outcome, ranged from 2.1 to 3.6 for the DOACs and 3.1 to 3.4 for warfarin across trials. ${ }^{1-3}$ Unlike the generally similar safety and effectiveness outcome rates, mortality rates in trials ${ }^{1-3,38}$ were one-fifth to one-third the mortality rates in the NH setting. Consistent with our findings, all-cause mortality rates were lower for the DOACs versus warfarin in trials ${ }^{1-3}$ and in Medicare. ${ }^{5}$ Other comparative studies among community-dwelling older adults which did not consider dosing have generally reported similar findings to the DOAC trials, with mixed signals of potential safety and effectiveness benefits for specific DOACs versus warfarin across studies. $^{4-6}$
Utilization of low and misaligned DOAC dosages was more prevalent in the $\mathrm{NH}$ than in community-based cohorts. ${ }^{8}, 39$ Although some fraction of such dosing is potentially inappropriate, deviation from labeled dosing is also likely to occur as a consequence of shared decision-making between clinicians, caregivers, and patients (as recommended in clinical guidelines ${ }^{40}$ ). Our results can be used as inputs into such shared decision-making processes, which should consider how safety and effectiveness of alternative medication and dosing regimens align with an individual's characteristics and goals of care. Dose-specific evidence on the safety and effectiveness of low DOAC dosages is limited, particularly 
among high-risk older adults. The $75 \mathrm{mg}$ dabigatran dose, which is only marketed in the USA, was not studied in the RE-LY trial. ${ }^{1}$ Only pooled analyses were reported for rivaroxaban doses in ROCKET-AF. ${ }^{2}$ Estimates for the $2.5 \mathrm{mg}$ apixaban dose were consistent with the $5 \mathrm{mg}$ dose in ARISTOTLE, although the sample size was small. ${ }^{3}$

Our findings were consistent with earlier research studying off-label dosing. Among community-dwelling patients, ischemic stroke rates were higher among low dose apixaban users with and without a renal indication. ${ }^{8}$ For apixaban users considered underdosed (i.e., $2.5 \mathrm{mg}$ without a renal indication), the ischemic stroke risk was nearly five times greater than among apixaban $5 \mathrm{mg}$ users, with similar bleeding rates. ${ }^{8}$ A pooled analysis of all 3 DOACs indicated that standard dosing in patients with a renal indication was associated with a more than twofold higher risk of major bleeding without a countervailing benefit for stroke risk. ${ }^{8}$ Another study of community-dwelling ORBIT-AF registry members reported similar off-label dosing-outcome associations. ${ }^{39}$
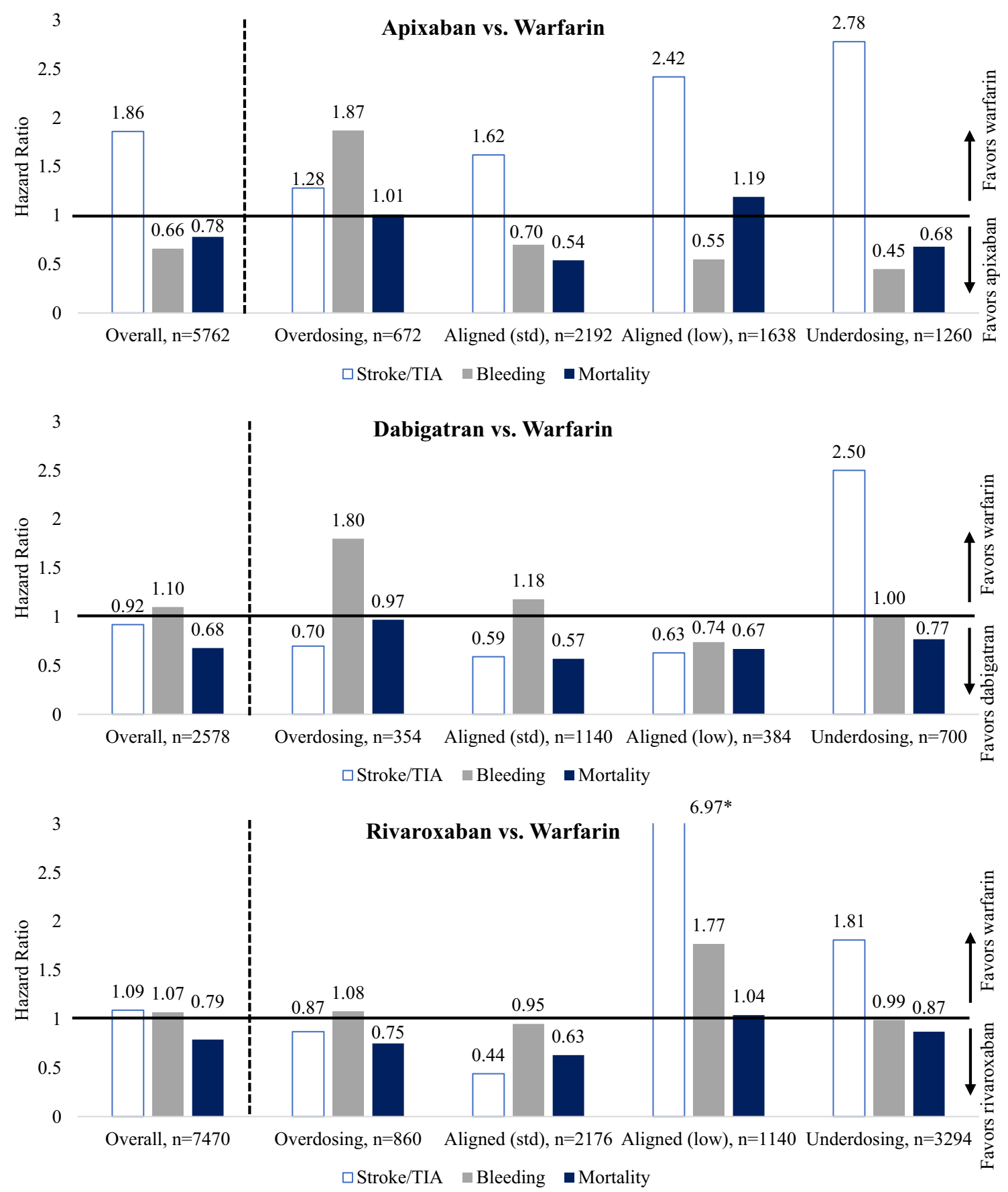

Figure 2 Results of Cox proportional hazards models comparing ischemic stroke/transient ischemic attack (TIA), bleeding, and mortality rates by dose between apixaban and warfarin users (top), dabigatran and warfarin users (middle), and rivaroxaban and warfarin users (bottom*). Asterisk indicates the axis for rivaroxaban versus warfarin was scaled to maintain consistency with the other plots. 
Low dose apixaban and rivaroxaban users (with or without an indication) had higher rates of ischemic stroke/TIA, while underdosing (but not low dosing by indication) of dabigatran was associated with elevated ischemic stroke/TIA risk. The increased bleeding rate among aligned low dose rivaroxaban (15 mg dose is $75 \%$ of the standard dose) but not underdosed rivaroxaban users may stem from the $36 \%$ of underdosed residents using rivaroxaban $10 \mathrm{mg}$ (50\% of standard) off-label. The protective association with mortality for underdosed DOACs, which was not present for aligned low dosages of apixaban or rivaroxaban, requires further study. Subtherapeutic dosing is expected to be associated with lower bleeding and higher ischemic event rates. Clinicians may have prescribed low dosages of DOACs for residents on the basis of unobserved perceived risk factors for bleeding or life-threatening bleeding. Because we lack cause of death information, under-detection of fatal clinical events was possible for residents who experienced a study outcome and were not hospitalized (i.e., died in the NH). However, a larger proportion of warfarin users that died were observed to have experienced a stroke, bleed, or other vascular event prior to death compared with users of each of the DOACs (Table 2). Comparatively lower fatal bleeding risk (versus similar warfarin users) that was of greater magnitude than any comparatively higher fatal ischemic event risk is one possible mechanism consistent with the observed mortality associations among the "underdosed" subgroup.

The recency of DOAC marketing and higher costs introduced the potential for channeling bias. ${ }^{35}$ Multiple studies in community-dwelling populations have found higher socioeconomic status to be associated with higher DOAC use after accounting for other factors (including prescriber specialty), ${ }^{41}$, 42 suggesting the cost of newer (branded) DOACs may have deterred utilization for certain patients of lower socioeconomic status. The effects of cost on treatment selection were less concerning in our study because more than two-thirds of DOAC and warfarin users were dually Medicare-Medicaid enrolled (and had copay assistance).

Changes in outcomes among residents initiating warfarin before and after DOACs were commonly used suggest the quality of warfarin therapy may have been shifting over time. Alternatively, those initiating warfarin after widespread DOAC use may have been better candidates for warfarin or residents of facilities with better management of warfarin patients. We lacked laboratory values on renal functioning and time in therapeutic range. The response to warfarin is varied and therefore warfarin therapy is titrated to a target range. Our comparative estimates represent the real-world outcomes for warfarin users based on how warfarin was managed in US nursing homes during the period 2011-2016. Systematic increases or decreases in the time spent in the therapeutic range among warfarin users in nursing homes would change the relative risk for adverse outcomes in DOAC versus warfarin comparisons. Minor adverse events managed in the $\mathrm{NH}$ were not detected and may have differed between groups; censoring for a treatment gap was more common among DOAC users and anticoagulant switching was more common among warfarin users. Such censoring may contribute to underestimation of the event rates that would have been observed had all residents remained on their index treatment. Generalizability to community-dwelling populations may be limited by distinct medication utilization patterns and goals of care in the NH setting.

\section{CONCLUSIONS}

DOAC users had lower mortality rates than warfarin users. Misaligned DOAC dosing was common (33.5\% apixaban, $40.9 \%$ dabigatran, $55.6 \%$ rivaroxaban) and less than recommended dosing was associated with higher ischemic cerebrovascular event rates and lower mortality rates (apixaban, dabigatran, and rivaroxaban) while higher than recommended dosing was associated with higher bleeding rates (apixaban and dabigatran). Ultimately, low ischemic stroke rates without excessive bleeding among both DOAC and warfarin users reinforce the clinical utility of anticoagulation with either class for older $\mathrm{NH}$ residents.

\section{Contributors: None.}

Corresponding Author: Matthew Alcusky, PharmD, PhD; Department of Population and Quantitative Health Sciences University of Massachusetts Medical School, Worcester, MA, USA (e-mail: matthew. alcusky@umassmed.edu).

Funding Information The study was funded by the National Institute on Aging (R21AG060529-01). DDM's time was also supported by RO1HL126911, RO1HL137734, RO1HL137794, RO1HL141434, RO1HL136660, and U54HL143541 from the National Heart, Lung, and Blood Institute.

\section{Compliance with ethical standards:}

Conflict of Interest: DDM has received research grant funding from Bristol-Myers Squibb, Boeringher-Ingelheim, Pfizer, Samsung, Philips Healthcare, Philips, Biotronik, and FlexCon and has received consultancy fees from Bristol-Myers Squibb, Pfizer, Flexcon, Boston Biomedical Associates. Other authors have no relationships to disclose.

\section{REFERENCES}

1. Connolly SJ, Ezekowitz MD, Yusuf S, et al. RE-LY Steering Committee and Investigators. Dabigatran versus warfarin in patients with atrial fibrillation. N Engl J Med. 2009;361:1139-51.

2. Patel MR, Mahaffey KW, Garg $\mathbf{J}$, et al. ROCKET AF Investigators. Rivaroxaban versus warfarin in nonvalvular atrial fibrillation. N Engl J Med. 2011;365:883-91.

3. Granger CB, Alexander JH, McMurray JJ, ARISTOTLE Committees and Investigators, et al.. Apixaban versus warfarin. $\mathrm{N}$ Engl $\mathrm{J}$ Med. 2011;365:981-992.

4. Coleman CI, Peacock WF, Bunz TJ, Alberts MJ. Effectiveness and Safety of Apixaban, Dabigatran, and Rivaroxaban Versus Warfarin in Patients with Nonvalvular Atrial Fibrillation and Previous Stroke or Transient Ischemic Attack. Stroke. 2017;48(8):2142-2149.

5. Lip GY, Keshishian A, Li X, et al. Effectiveness and Safety of Oral Anticoagulants Among Nonvalvular Atrial Fibrillation Patients. Stroke. 2018;49(12):2933-2944. 
6. Martinez BK, Sood NA, Bunz TJ, Coleman CI. Effectiveness and Safety of Apixaban, Dabigatran, and Rivaroxaban Versus Warfarin in Frail Patients With Nonvalvular Atrial Fibrillation. J Am Heart Assoc. 2018;7: e008643.

7. Alcusky M, McManus DD, Hume AL, Fisher M, Tjia J, Lapane KL. Changes in Anticoagulant Utilization among United States Nursing Home Residents with Atrial Fibrillation from 2011-2016. J Am Heart Assoc. 2019;7(9):e012023

8. Yao X, Shah ND, Sangaralingham LR, Gersh BJ, Noseworthy PA. NonVitamin K Antagonist Oral Anticoagulant Dosing in Patients With Atrial Fibrillation and Renal Dysfunction. J Am Coll Cardiol. 2017;69:27792790 .

9. Saliba S, Buchcanan J. Development \& Validation of a Revised Nursing Home Assessment Tool: MDS 3.0. Rand Health Corporation. 2008. Centers for Medicare and Medicaid Services Contract No. 500-00-0027.

10. Cunningham A, Stein CM, Chung CP, Daugherty JR, Smalley WE, Ray WA. An automated database case definition for serious bleeding related to oral anticoagulant use. Pharmacoepidemiol Drug Saf. 2011;20(6):560-6.

11. White RH, Garcia M, Sadeghi B, et al. Evaluation of the predictive value of ICD-9-CM coded administrative data for venous thromboembolism in the United States. Thromb Res. 2010;126(1):61-67.

12. Kumamaru H, Judd SE, Curtis JR, et al. Validity of claims-based stroke algorithms in contemporary medicare data: reasons for geographic and racial differences in stroke (REGARDS) study linked with medicare claims. Circ Cardiovasc Qual Outcomes. 2014;7:611-9.

13. Kiyota Y, Schneeweiss S, Glynn RJ, Cannuscio CC, Avorn J, Solomon DH. Accuracy of medicare claims-based diagnosis of acute myocardial infarction: Estimating positive predictive value on the basis of review of hospital records. Am Heart J. 2004; 148(1):99-104.

14. Centers for Medicare and Medicaid Services (CMS). ICD-10-CM and GEMs. 2016. from https://www.cms.gov/Medicare/Coding/ICD10/ 2016-ICD-10-CM-and-GEMs.html. Accessed September 5, 2018

15. Lip GYH, Nieuwlaat R, Pisters R, Lane DA, Crijns HJGM. Refining clinical risk stratification for predicting stroke and thromboembolism in atrial fibrillation using a novel risk factor-based approach: The Euro Heart Survey on atrial fibrillation. Chest. 2010;137(2):263-272.

16. Fang MC, Go AS, Chang $\mathbf{Y}$, et al. A new risk scheme to predict warfarinassociated hemorrhage: The ATRIA (Anticoagulation and Risk Factors in Atrial Fibrillation) Study. J Am Coll Cardiol. 2011;58:395-401.

17. Bangalore S, Fakheri R, Toklu B, Ogedegbe G, Weintraub H, Messerli FH. Angiotensin-Converting Enzyme Inhibitors or Angiotensin Receptor Blockers in Patients Without Heart Failure? Insights From 254,301 Patients From Randomized Trials. Mayo Clin Proc. 2016;91(1):51-60.

18. Coxib and traditional NSAID Trialists' (CNT) Collaboration, Bhala N, Emberson J, et al. Vascular and upper gastrointestinal effects of non steroidal anti-inflammatory drugs: meta-analyses of individual participant data from randomised trials. Lancet. 2013;382(9894):769-79.

19. Quinn GR, Singer DE, Chang Y, et al. Effect of selective serotonin reuptake inhibitors on bleeding risk in patients with atrial fibrillation taking warfarin. Am J Cardiol. 2014;114:583-6.

20. Dans AL, Connolly SJ, Wallentin L, et al. Concomitant use of antiplatelet therapy with dabigatran or warfarin in the Randomized Evaluation of Long-Term Anticoagulation Therapy (RE-LY) trial. Circulation. 2013;127(5):634-40.

21. Yebyo HG, Aschmann HE, Kaufmann M, Puhan MA. Comparative effectiveness and safety of statins as a class and of specific statins for primary prevention of cardiovascular disease: A systematic review, metaanalysis, and network meta-analysis of randomized trials with 94,283 participants. Am Heart J. 2019;210:18-28.

22. Winkelmayer WC, Schneeweiss S, Mogun H, Patrick AR, Avorn J, Solomon DH. Identification of individuals with CKD from medicare claims data: A validation study. Am J Kidney Dis. 2005;46(2):225-232.

23. Thomas KS, Dosa D, Wysocki A, Mor V. The Minimum Data Set 3.0 Cognitive Function Scale. Med Care. 2017;55(9):e68-e72.

24. Morris J, Fries B, Morris S. Scaling ADLs with the MDS. J Gerontol A Biol Sci Med Sci. 1999;54(11):M546-M553.

25. Kurichi JE, Bogner HR, Streim JE, et al. Predicting 3-year mortality and admission to acute-care hospitals, skilled nursing facilities, and longterm care facilities in Medicare beneficiaries. Arch Gerontol Geriatr. 2017;73:248-256.
26. Hapca S, Guthrie B, Cvoro V, et al. Mortality in people with dementia, delirium, and unspecified cognitive impairment in the general hospital: prospective cohort study of 6,724 patients with 2 years follow-up. Clin Epidemiol. 2018;10:1743-1753.

27. Leelakanok $\mathbf{N}$, Holcombe $\mathbf{A L}$, Lund BC, Gu X, Schweizer ML Association between polypharmacy and death: A systematic review and meta-analysis. J Am Pharm Assoc (2003). 2017;57(6):729-738.e10.

28. Wyss R, Girman CJ, LoCasale RJ, Brookhart AM, Stürmer T. Variable Selection for Propensity Score Models When Estimating Treatment Effects on Multiple Outcomes: a Simulation Study. Pharmacoepidemiol Drug Saf. 2013;22(1):77-85

29. Patrick AR, Schneeweiss S, Brookhart MA, et al. The implications of propensity score variable selection strategies in pharmacoepidemiology: an empirical illustration. Pharmacoepidemiol Drug Saf. 2011;20(6):551-

30. Brookhart MA, Schneeweiss S, Rothman KJ, Glynn RJ, Avorn J, Stürmer T. Variable selection for propensity score models. Am J Epidemiol. 2006;163(12):1149-1156.

31. Ali MS, Groenwold RH, Pestman WR, et al. Propensity score balance measures in pharmacoepidemiology: a simulation study. Pharmacoepidemiol Drug Saf. 2014;23(8):802-11.

32. Pradaxa [package insert]. Ridgefield, CT: Boehringer Ingelheim. 2012 https: / /www.accessdata.fda.gov/drugsatfda_docs/label/2010/ 022512s000lbl.pdf. Accessed June 25, 2017.

33. Xarelto [package insert]. Leverkusen, Germany: Janssen. 2011. https:// www.accessdata.fda.gov/drugsatfda_docs/label/2011/022406s000lbl. pdf. Accessed June 25, 2017.

34. Eliquis [package insert]. Princeton, New Jersey: Bristol Myers Squibb. 2012. https://www.accessdata.fda.gov/drugsatfda_docs/label/2012/ 202155s000lbl.pdf. Accessed September 1, 2017.

35. Petri H, Urquhart $\mathbf{J}$. Channeling bias in the interpretation of drug effects. Stat Med. 1991;10(4):577-81.

36. Yale New Haven Health Services Corporation/Center for Outcomes Research \& Evaluation. 2014 Measures updates and specifications report hospital-level 30-day risk-standardized readmission measures. 2014. Available at: http://altarum.org/sites/default/files/uploaded-publicationfiles/Rdmsn_Msr_Updts_HWR_0714_0.pdf. Accessed February 27, 2019.

37. Lindenauer PK, Normand SLT, Drye EE, et al. Development, validation, and results of a measure of 30-day readmission following hospitalization for pneumonia. J Hosp Med. 2011;6(3):142-150.

38. Mant J, Hobbs F, Fletcher $\mathbf{K}$, et al. Warfarin versus aspirin for stroke prevention in an elderly community population with atrial fibrillation: a randomized controlled trial. Lancet. 2007;370:493-503.

39. Steinberg BA, Shrader $\mathbf{P}$, Thomas $\mathbf{L}$, et al. ORBIT-AF Investigators and Patients. Off-Label Dosing of Non-Vitamin K Antagonist Oral Anticoagulants and Adverse Outcomes: The ORBIT-AF II Registry. J Am Coll Cardiol. 2016;68(24):2597-2604.

40. January CT, Wann LS, Calkins H, et al. 2019 AHA/ACC/HRS Focused Update of the 2014 AHA/ACC/HRS Guideline for the Management of Patients With Atrial Fibrillation: A Report of the American College of Cardiology/American Heart Association Task Force on Clinical Practice Guidelines and the Heart Rhythm Society. J Am Coll Cardiol. 2019 (19)30209-8.

41. Zhu J, Alexander GC, Nazarian S, Segal JB, Wu AW. Trends and Variation in Oral Anticoagulant Choice in Patients with Atrial Fibrillation, 2010-2017. Pharmacotherapy. 2018;38(9):907-920.

42. Steinberg BA, Shrader P, Thomas L, et al. Outcomes Registry for Better Informed Treatment of Atrial Fibrillation (ORBIT-AF) Investigators and Patients. Factors associated with non-vitamin K antagonist oral anticoagulants for stroke prevention in patients with new-onset atrial fibrillation: Results from the Outcomes Registry for Better Informed Treatment of Atrial Fibrillation II (ORBIT-AF II). Am Heart J. 2017;189:40-47.Publisher's Note Springer Nature remains neutral with regard to jurisdictional claims in published maps and institutional affiliations.

Publisher's Note: Springer Nature remains neutral with regard to jurisdictional claims in published maps and institutional affiliations. 